\title{
Population pharmacokinetics of nevirapine in an unselected cohort of HIV-I-infected individuals
}

\author{
Monique M. R. de Maat,' Alwin D. R. Huitema,' Jan W. Mulder, ${ }^{2}$ Pieter L. Meenhorst, ${ }^{2}$ \\ Eric C. M. van Gorp ${ }^{2}$ \& Jos H. Beijnen ${ }^{1,3}$ \\ ${ }^{1}$ Slotervaart Hospital, Department of Pharmacy \& Pharmacology, ${ }^{2}$ Department of Internal Medicine, Amsterdam and ${ }^{3}$ University of Utrecht, Faculty of \\ Pharmacy, Utrecht, The Netherlands
}

\begin{abstract}
Aims To study the population pharmacokinetics of nevirapine and to identify relationships between patient characteristics and pharmacokinetics in an unselected population of patients attending our outpatient clinic.

Methods Ambulatory HIV-1-infected patients from the outpatient clinic of the Slotervaart Hospital who were being treated with a nevirapine-containing regimen were included. During each visit, blood samples were collected for the determination of nevirapine plasma concentrations and clinical chemistry parameters. Variables that were collected at baseline were serology for hepatitis B (HBV) and C (HCV) viruses, liver enzymes, and total bilirubin (TBR). In addition, information about concomitant use of St John's wort and patient demographics were included. The pharmacokinetics of nevirapine were described by first-order absorption and elimination using nonlinear mixed effect modelling (NONMEM V1.1). Population pharmacokinetic parameters (apparent clearance $(\mathrm{CL} / F)$, volume of distribution $(V /$ $F$ ), absorption rate constant $\left(k_{\mathrm{a}}\right)$ ) were estimated, as were interindividual, interoccasion, and residual variability in the pharmacokinetics. The influence of patient characteristics on the pharmacokinetics of nevirapine was determined.

Results From 173 outpatients a total number of 757 nevirapine plasma concentrations at a single random time point and full pharmacokinetic curves for 13 patients were available resulting in a database of 1329 nevirapine plasma concentrations. Mean CL/F, V/F, and $k_{\mathrm{a}}$ were $3.271 \mathrm{~h}^{-1}, 106 \mathrm{l}$, and $01.66 \mathrm{~h}^{-1}$, respectively. CL/F of nevirapine was correlated with weight, chronic HCV infection, and baseline aspartate aminotransferase (ASAT). Chronic HCV and baseline ASAT $>1.5 \times$ upper limit of normal (ULN) decreased CL/F by $27.4 \%$ and $13.2 \%$, respectively, whereas an increase in body weight of $10 \mathrm{~kg}$ increased $\mathrm{CL} / \mathrm{F}$ by $0.141 \mathrm{~h}^{-1}$. A trend towards a lower $\mathrm{CL} / F$ in patients of the Negroid race was observed. No significant covariates were found for $V / F$.

Conclusions The pharmacokinetics of nevirapine were adequately described by our population pharmacokinetic model. Weight, chronic HCV infection, and baseline ASAT were found to be significant covariates for CL/F of nevirapine. The model incorporating these significant covariates may be an important aid in further optimizing nevirapine-containing therapy.
\end{abstract}

Keywords: nevirapine, patient characteristics, population pharmacokinetics

\section{Introduction}

Nevirapine $\left(\right.$ Viramune $\left.^{\circledR}\right)$ is a non-nucleoside reverse transcriptase inhibitor (NNRTI), which is indicated for use

Correspondence: M. M. R. de Maat,. PharmD, Slotervaart Hospital, Department of Pharmacy \& Pharmacology, Louwesweg 6, 1066 ec Amsterdam, The Netherlands. Tel.: (+)3 I-20-5 I2448I; Fax: (+)3I 20 5I24753; E-mail: apmod@slz.nl

Accepted 22 July 2002. in the treatment of human immunodeficiency virus type 1 (HIV-1) infection. The drug binds directly to the viral reverse transcriptase to block polymerase activity by causing a disruption of the catalytic site [1]. The licensed dosage of nevirapine in HIV-1-infected adults is $200 \mathrm{mg}$ once daily during the first 2 weeks, followed by $200 \mathrm{mg}$ twice daily thereafter [2]. However, it is also used in a once daily dosing regimen of $400 \mathrm{mg}$ [3].

The pharmacokinetics of nevirapine are characterized 
by rapid and nearly complete oral absorption, rapid distribution, and prolonged elimination [4]. In vitro studies using human liver microsomes demonstrated that CYP3A is primarily responsible for the oxidative metabolism of nevirapine to its major (inactive) metabolites, although other CYP enzymes may have a secondary role [5]. Nevirapine metabolism is subject to autoinduction, resulting in a decrease in the terminal half-life from $45 \mathrm{~h}$ following a single dose to approximately $25-30 \mathrm{~h}$ after multiple dosing [1]. The urinary excretion of nevirapine is minor, since only a small fraction $(<3 \%)$ is eliminated as parent compound in urine [1].

Although nevirapine, when used in combination therapy, has proven to be safe and effective [6,7], highly drug-resistant virus rapidly emerges when administered in suboptimal regimens [8]. Havlir et al. reported a correlation between plasma trough concentrations of nevirapine and the virological response in HIV-1infected patients [9]. Furthermore, an analysis of plasma concentrations obtained in the INCAS Study showed that a high exposure is associated with improved virological response in the short as well as in the long term [10]. In contrast, a relationship between plasma concentration of nevirapine and toxicity has not been established conclusively.

Interpatient variability may be influenced by many factors such as comedication, comorbidity, etc. Knowledge of such factors could be very useful in dose individualization when a target plasma concentration of nevirapine has been defined. Zhou et al. demonstrated that the apparent clearance $(\mathrm{CL} / F)$ of nevirapine was correlated with gender, with a typical value of 3.971 $\mathrm{h}^{-1}$ for men compared with $3.021 \mathrm{~h}^{-1}$ for women [11]. In addition, severe hepatic dysfunction has been shown to increase exposure to nevirapine substantially [12]. Thus far, no other patient characteristics have been identified that could explain the interpatient variability in the pharmacokinetics of nevirapine.

The objective of this study was to develop a population pharmacokinetic model for nevirapine and to identify the relationships between patient characteristics and pharmacokinetics in a representative patient population attending a routine outpatient HIV clinic.

\section{Methods}

\section{Patients}

Ambulatory HIV-1-infected patients from the outpatient clinic of the Slotervaart Hospital, Amsterdam, The Netherlands, were recruited. Data were collected retrospectively from January 1997 to April 2000. All patients were using nevirapine as part of their antiretroviral regimen and had at least one nevirapine plasma sample available for analysis. No other restrictions were made for inclusion into this study.

Approval was obtained from the institutional review board of Slotevaart Hospital and all patients gave written informed consent.

\section{Sampling and bioanalysis}

At each visit to the clinic, a single blood sample was obtained for the determination of nevirapine concentration. In our hospital, a strict protocol is utilized in which plasma concentrations of antiretroviral drugs are routinely and frequently measured within a therapeutic drug monitoring (TDM)-program. Therefore, patients are familiar with the need to remember the time of ingestion of the last dose. Additionally, sampling times are recorded electronically in the Department of Clinical Chemistry. Time after ingestion is extracted from this information. Cases where plasma concentration was determined to check compliance were not included in this analysis. In addition to the sparse randomly timed samples, full pharmacokinetic curves from 13 patients were available, obtained as part of another study [3]. All nevirapine plasma concentrations were determined at steady-state. Concentrations of nevirapine were measured by a validated high-performance liquid chromatographic method (h.p.l.c.) [13] which was validated over the range of $0.05-10 \mu \mathrm{g} \mathrm{ml}^{-1}$ using $250 \mu \mathrm{l}$ of plasma. Recovery of nevirapine from human plasma was $94.5 \%$. Within- and between-day coefficients of variation were less than $4.5 \%$ for all quality control samples covering the complete calibration curve. The corresponding accuracies ranged from 97.3 to $105.2 \%$.

\section{Pharmacokinetic analyses}

All analyses were performed with the nonlinear mixedeffect modelling program (NONMEM) version $\mathrm{V}$ (double precision, level 1.1) [14]. The first-order conditional estimation (FOCE) method was used throughout. The performance of the tested models was assessed by both statistical and graphical methods. The minimal value of the objective function (equal to minus twice the $\log$ likelihood) as calculated by NONMEM was one of the parameters used to assess goodness-of-fit. Discrimination between hierarchical models was based on this value using the $\log$ likelihood ratio test [14]. Standard errors for all parameters were calculated using the COVARIANCE option of NONMEM and individual Bayesian pharmacokinetic parameters were obtained with the POSTHOC option [14]. For graphical model diagnostics, the S-plus (MathSoft Inc, Seattle, USA) based model building aid Xpose 3.0 was used [15]. 


\section{Basic pharmacokinetic model}

An open one-compartment model with first-order elimination was used to describe the data. Both zero-order and first-order absorption models with and without absorption lag-time were tested. Furthermore, different time-dependent absorption models as described by Higaki et al. were tested [16]. Also, a two-compartment model and Michaelis Menten-elimination were tested.

Interindividual variability in the different pharmacokinetic parameters was estimated with a proportional error model. Interoccasion (intraindividual) variability was also estimated with a proportional model as suggested by Karlsson \& Sheiner [17]. For instance, variability in $\mathrm{CL} / F$ was estimated using CL/ $F_{\mathrm{ij}}=\theta^{\star}\left(1+\eta_{\mathrm{i}}+\kappa_{\mathrm{j}}\right)$, in which $\mathrm{CL} / F_{\mathrm{ij}}$ represents the apparent clearance of the $\mathrm{i}^{\text {th }}$ individual at the $\mathrm{j}^{\text {th }}$ occasion, $\theta$ is the typical value of $\mathrm{CL} / F$ in the population, $\eta_{\mathrm{i}}$ is the interindividual random effect with mean 0 and variance $\omega^{2}$ and $\kappa_{\mathrm{j}}$ is the interoccasion random effect with mean 0 and variance $\pi^{2}$. Covariance between differents (interindividual variability) was tested. Also covariance between different kappas (interoccasion variability) was tested. This latter covariance was modelled as $P_{j}=\theta_{1} \star\left(\kappa_{1 j}+\theta_{2}{ }^{\star} \kappa_{2 j}\right)$, in which $P_{j}$ is the value of parameter $P$ at occasion $j$, $\kappa_{1 j}$ is the interoccasion random effect of parameter $1, \kappa_{2 j}$ is the interoccasion random effect of parameter 2 and $\theta_{2}$ is the correlation factor between these interoccasion effects.

Residual variability was estimated with an additive error model. Since larger residual variability was likely to be associated with samples collected closer to the time of intake, for instance because of irregular absorption, a mixture additive error model was used to describe residual variability. This model is represented by:

$$
C_{\mathrm{ij}}=\hat{C}_{\mathrm{ij}}+\theta_{\mathrm{ip}} \times \varepsilon_{\mathrm{ij}}
$$

in which $C_{\mathrm{ij}}$ and $\hat{\mathrm{C}}_{\mathrm{ij}}$ are, respectively, the $\mathrm{i}^{\text {th }}$ measured and model predicted drug concentration of the $\mathrm{j}^{\text {th }}$ individual, $\epsilon_{\mathrm{ij}}$ is the residual random error with mean 0 and variance $\sigma^{2}$ and $\theta_{\text {ip }}$ is an increment proportion (ip), whose value is to be estimated but fixed to 1 for time points exceeding $2 \mathrm{~h}$ after intake. The value of $\sigma^{2}$ may vary between individuals. Therefore the assumption of a constant $\sigma^{2}$ for all individuals may result in bias. In order to reduce this bias, two populations with different values for $\sigma^{2}$ were assumed to exist. Therefore residual error parameters estimated were the fraction of patients in population 1 , residual variability of population 1 and 2 and the increment proportion.

\section{Covariate model building}

In order to establish possible relationships between the pharmacokinetics of nevirapine and patient characteristics, the following covariates were collected at baseline: gender (SEX), race (RACE), chronic hepatitis C (HCV) and/or chronic hepatitis B (HBV) status, and liver enzymes (LE) including alanine aminotransferase (ALAT,

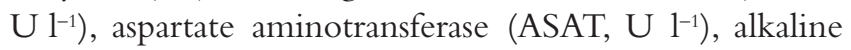
phosphatase (AP, $\left.\quad \mathrm{U}^{-1}\right)$, gamma-glutamyltransferase (GGT, $\mathrm{U} \mathrm{l}^{-1}$ ), and serum total bilirubin (TBR, $\mu \mathrm{mol} \mathrm{l}^{-1}$ ). In addition, covariates determined during treatment with nevirapine were collected and included age (AGE, years), body weight (WT, kg), concomitant use of St John's wort, serum glucose (GLU, mmol $\left.\mathrm{l}^{-1}\right)$, serum creatinine (KR, mmol $\mathrm{l}^{-1}$ ), serum albumin (ALB, $\mathrm{g}^{-1}$ ), serum total protein (TP, $\left.\mathrm{g}^{-1}\right)$, amylase (AM, $\left.\mathrm{U}^{-1}\right)$, and creatine kinase $\left(\mathrm{CK}, \mathrm{U}^{-1}\right)$. The constituents, quality, and dose of St John's wort were not taken into account because of too few patients using this agent concomitantly. Patients were taking few other drugs with their antiretroviral therapy. In addition, little or no effect on the pharmacokinetics of nevirapine by coadministered drugs has been reported. The number of patients who were taking specific additional drugs was too small to include in this analysis. Therefore, no other concomitantly used drugs were included as a covariate. AGE, GLU, KR, ALB, TP, $\mathrm{AM}, \mathrm{CK}$, and WT were examined as continuous variables. Sex, Race, St John's Wort, HCV, and HBV were examined as dichotomous variables. The absolute baseline LEs (ALAT, ASAT, AP, GGT) and TBR were also transformed to dichotomous variables (below or exceeding 1.5 times the upper limit of normal (ULN)). In the light of the recommendations of the European Medicines Evaluation Agency (EMEA), the choice of a breakpoint of $1.5 \times$ ULN was made. It is unlikely that patients with high LEs will start nevirapine-containing regimens in the future. From a small fraction of patients, baseline LEs and TBR were not available. In order to avoid bias, a covariate was included in the model indicating missing data. For instance, the influence of a dichotomous covariate $\mathrm{X}$ on $\mathrm{CL} / F$ with missing data of $\mathrm{X}$ for some individuals was modelled as TVCL $=\theta_{1} \times \theta_{2}{ }^{\mathrm{X}} \times(1-\mathrm{MISS}) \times \theta_{3}{ }^{\text {MISS }}$, in which TVCL is the typical value of CL/F in the population, MISS is 1 for records with missing data and 0 for all other records, $\theta_{1}$ is the typical value of an individual with $X=0$ (no missing data), $\theta_{2}$ is the relative difference in $\mathrm{CL} / F$ for individuals with $\mathrm{X}=1$ (no missing data) and $\theta_{3}$ is the relative difference in CL/F for individuals with missing data.

A covariate was included in an intermediate model when inclusion of this covariate was both statistically significant and relevant. A covariate was considered statistically significant when inclusion of the covariate was associated with a decrease in minimal value of the objective function associated with a $P$ value of $\leq 0.05$ (log-likelihood ratio test). Relevance was reached when the typical value of the pharmacokinetic parameter of interest changed at least $10 \%$ within the observed range of that covariate in the population. 
All significant and relevant covariates were included in an intermediate model. Finally, a stepwise backward elimination procedure was carried out. Again, a parameter was only retained in the model when the influence of this parameter was statistically significant (log-likelihood ratio test: $P \leq 0.05$ ) and relevant (as defined earlier).

The actual significance level of the covariates included into the model was assessed with a randomization test, where a large number of datasets are created that could have arisen under the null hypothesis (i.e. the covariate is not related to the pharmacokinetic parameter). These datasets are created by random permutation of the empirical distribution of the covariate in the original dataset. The final model is applied to these datasets. The distribution of the difference in objective function between the models with and without the (randomly permutated) covariate is obtained and the actual significance level (one-sided test) can be calculated. In this validation, at least 1000 datasets were created for all covariates. Wings for NONMEM (version 300) was used to perform these tests [18].

\section{Results}

\section{Patients}

Data from 173 HIV-1-infected patients were available and clinical details are presented in Table 1. The median age was 41.5 years (interquartile range (IQR) 36.1-48.2), and median body weight was $72 \mathrm{~kg}$ (IQR 65-79). The patient population was predominantly male $(89.6 \%)$ and Caucasian (89\%). Five patients were concomitantly using St John's wort. Baseline LEs were not available in 3 to $13 \%$ of patients (depending on the specific enzyme). A total number of 757 nevirapine plasma concentrations at a single random time point and full pharmacokinetic profiles from 13 patients (two curves per patient consisting of 17 and 27 time points) were available, resulting in a database of 1329 nevirapine plasma concentrations. Excluding the data from the full pharmacokinetic curves,

Table 1 Characteristics of 173 HIV type 1 infected individuals enrolled in this study of the population pharmacokinetic analysis of nevirapine.

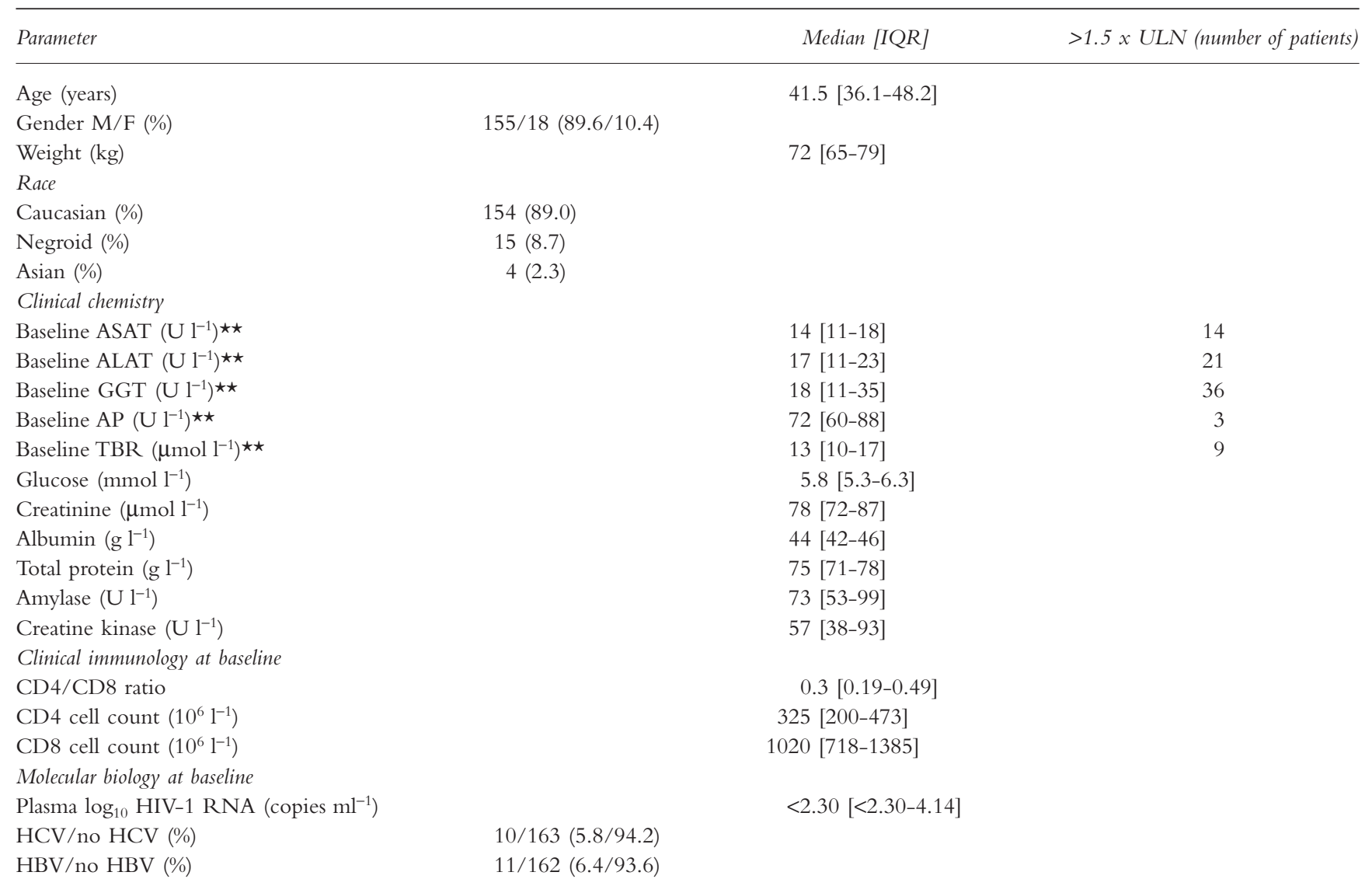

ALAT $=$ alanine aminotransferase, $\mathrm{AP}=$ alkaline phosphatase, $\mathrm{ARV}=$ antiretroviral, $\mathrm{ASAT}=$ aspartate aminotransferase, $\mathrm{F}=\mathrm{female}, \mathrm{GGT}=$ gammaglutamyltransferase, $\mathrm{HBV}=$ hepatitis $\mathrm{B}$ infection, $\mathrm{HCV}=$ hepatitis $\mathrm{C}$ infection, $\mathrm{IQR}=$ interquartile range, $\mathrm{M}=\mathrm{male}, \mathrm{PI}=$ protease inhibitor, TBR $=$ Total bilirubin, ULN $=$ Upper limit of normal.

${ }^{\star *} \operatorname{ASAT} n=167$, ALAT $n=167$, GGT $n=150$, AP $n=166$, TBR $n=164$. 
Table 2 Final parameter estimates of basic and final pharmacokinetic model.

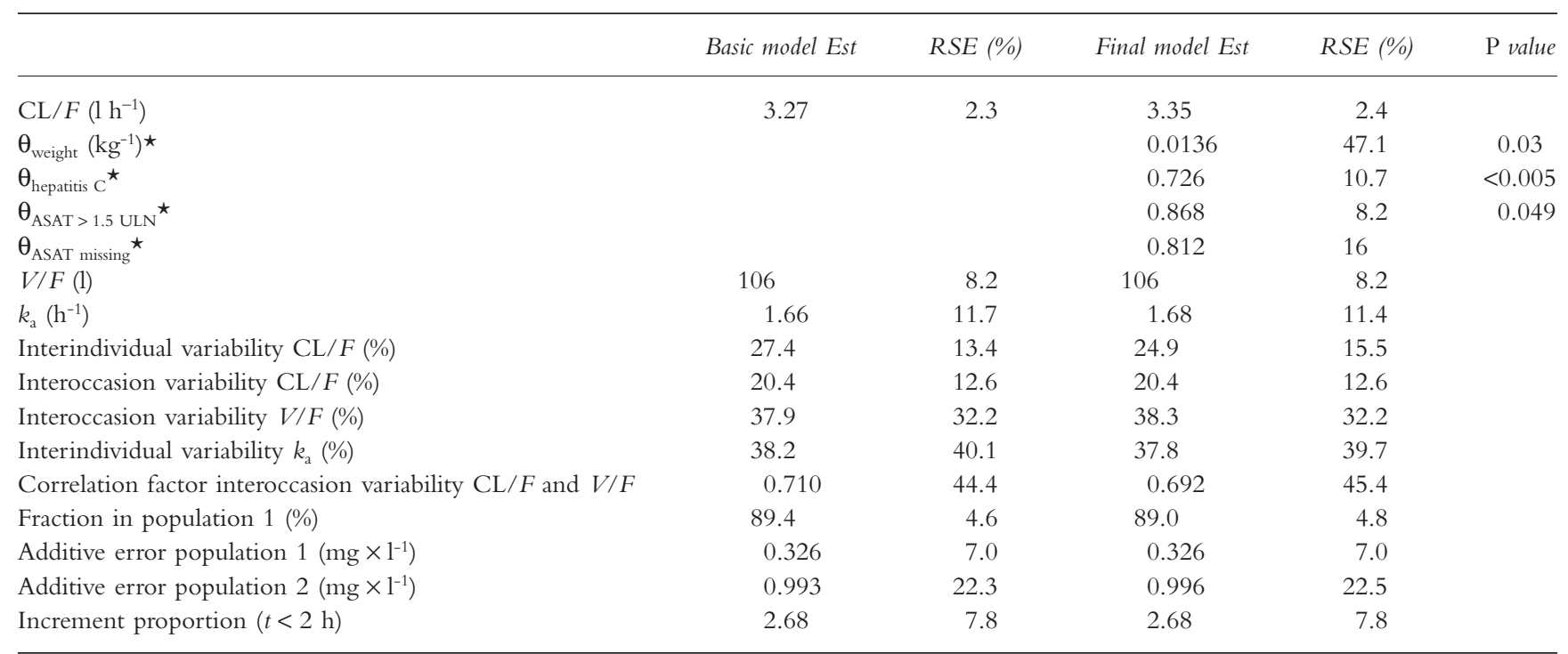

ASAT $=$ aspartate aminotransferase, $\mathrm{CL} / F=$ apparent clearance, Est $=$ parameter estimate, $k_{\mathrm{a}}=$ absorption rate constant, RSE $=$ relative standard error (as calculated with COVARIANCE option of NONMEM), $V / F=$ volume of distribution; $P$ value estimated with randomization test.

${ }^{\star} \mathrm{CL} / F=\theta_{\mathrm{CL} / F} \mathrm{X}\left(1+\theta_{\text {weight }}[\mathrm{WT}-70]\right) \times\left(\theta_{\text {hepatitis } \mathrm{C}}\right)^{\mathrm{HCV}} \times\left(\theta_{\mathrm{ASAT}>1.5 \mathrm{xULN}}\right)^{(1-\mathrm{MISS}) \times \mathrm{ASAT}} \times\left(\theta_{\mathrm{ASAT} \text { missing }}\right)^{\mathrm{MISS}}$, in which WT is weight, HCV is 1 for individuals with chronic hepatitis $\mathrm{C}$ infection and 0 for all others, ASAT is 1 for patients with baseline ASAT $>1.5 \times$ ULN and 0 for all others, and MISS is 1 for patients with no baseline ASAT value and 0 for all others.

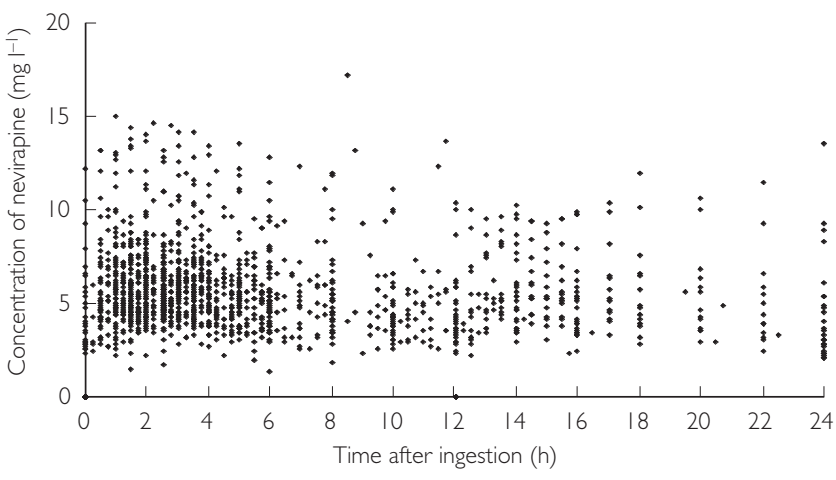

Figure 1 Concentration-time data for nevirapine in a randomly selected population of HIV-1 infected patients.

a mean of 4-5 samples per patient (range 1-10) was used. Figure 1 shows the nevirapine concentration-time data.

\section{Pharmacokinetics}

The population pharmacokinetics of nevirapine were best described by a one-compartment model with firstorder absorption and elimination. The results of parameters generated from this model are summarized in Table 2. The use of absorption lag-time or timedependent absorption models did not increase the goodness-of-fit, neither did that of a two-compartmentmodel or one with Michaelis Menten-elimination. Estimates of $\mathrm{CL} / F, V / F$ and $k_{\mathrm{a}}$ were $3.271 \mathrm{~h}^{-1}, 1061$ and $1.66 \mathrm{~h}^{-1}$, respectively. Interindividual variability in $V / F$ and interoccasion variability in $k_{\mathrm{a}}$ could not be estimated.
Furthermore, the inclusion of the correlation factor between interoccasion variability of $\mathrm{CL} / F$ and $V / F$ resulted in an increased goodness-of-fit. Figure 2 shows the predicted concentrations from the basic model vs observed concentrations of nevirapine (Panel a). The inclusion of two populations with different residual errors and the introduction of an increment proportion $\left(\theta_{\mathrm{ip}}\right)$ both resulted in a dramatic increase in goodness-of-fit $(\Delta$ Objective function -300 and -200 , respectively). A large fraction of the population (89.4\%) was associated with a relatively small additive error of $0.326 \mathrm{mg} \mathrm{l}^{-1}$, whereas the other fraction was associated with a larger additive error of $0.996 \mathrm{mg} \mathrm{l}^{-1}$. The residual variability in the samples collected until $2 \mathrm{~h}$ after ingestion was estimated to be 2.7-fold higher than samples collected after this period (Table 2).

The different covariates were introduced separately into the basic model for $\mathrm{CL} / F$ and $V / F$. SEX, WT, HCV, ALAT, ASAT, TBR, and RACE had a relevant relationship with $\mathrm{CL} / F$, which was associated with a significant and relevant increase in goodness-of-fit. For $V / F$, none of the covariates was significantly correlated. A step-wise backward elimination was then performed to eliminate nonsignificant and nonrelevant covariates from the intermediate model to develop the final model. Only WT, HCV, and ASAT had a significant and relevant relationship with $\mathrm{CL} / F$, although RACE was of borderline significance $(P$ value log-likelihood ratio test $=0.055)$. Subsequently, a randomization test was performed, which yielded a $P$ value of 0.058 and, conse- 

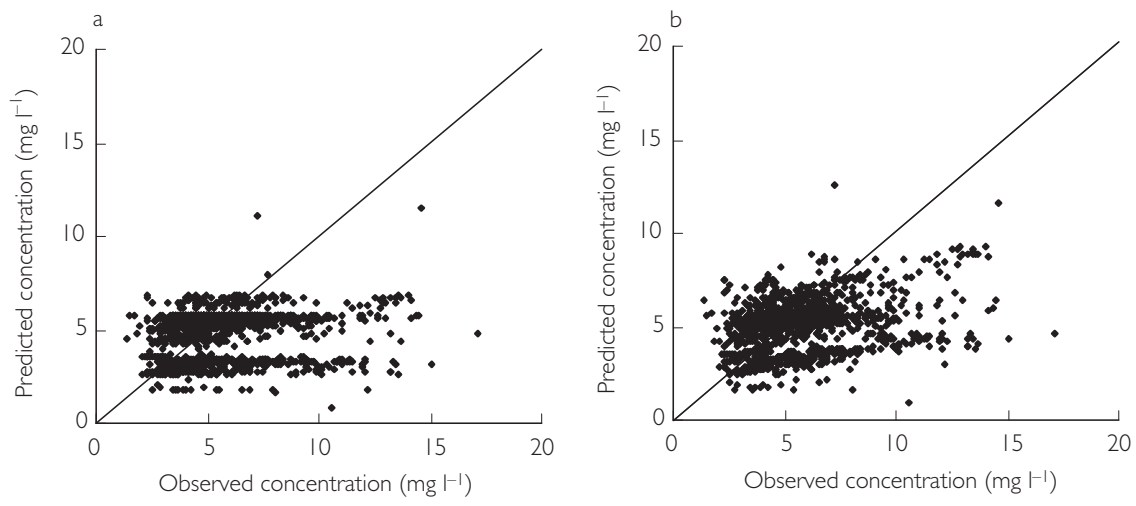

Figure 2 Model predicted $v$ s observed concentrations of nevirapine using the basic model (Panel a) and the final model (Panel b).

quently, RACE was removed from the final model. The results of the final pharmacokinetic model are presented in Table 2. Figure 2 (Panel b) shows the model predicted concentration $v s$ the observed concentration of nevirapine using the final model.

The results of the randomization test applied to the final model showed that WT, HCV, and ASAT were significantly related to the CL/F of nevirapine (Table 2 ). As shown in Figure 2, the final model (Panel b) describes the data better than the basic model (Panel a). The magnitudes of reduction in CL/ $F$ were $27.4 \%$ and $13.2 \%$ for $\mathrm{HCV}$ and ASAT $>1.5 \times \mathrm{ULN}$, respectively, whereas an increase of $10 \mathrm{~kg}$ in WT increases the $\mathrm{CL} / F$ by $0.141 \mathrm{~h}^{-1}$. The following equation describes the final model for $\mathrm{CL} / F$ :

$$
\begin{aligned}
\mathrm{CL} / F= & (3.35+0.0136 \times[\mathrm{WT}-70]) \times 0.726^{\mathrm{HCV}} \\
& \times 0.868^{\mathrm{ASAT}} \times(1-\mathrm{MISS}) \times 0.812^{\mathrm{MISS}}
\end{aligned}
$$

in which HCV is 1 for individuals with chronic hepatitis $\mathrm{C}$ infection and 0 for all others, ASAT is 1 for patients with baseline ASAT $>1.5 \times$ ULN and 0 for all others, and MISS is 1 for patients with no baseline ASAT value and 0 for all others.

\section{Discussion}

The population pharmacokinetics of nevirapine were best described by a one-compartment model with firstorder absorption and elimination. Application of the basic pharmacokinetic model to the complete data set resulted in typical values of CL/F and $V / F$ of $3.271 \mathrm{~h}^{-1}$ and 1061 , respectively. That for $k_{\mathrm{a}}$ was high $\left(1.66 \mathrm{~h}^{-1}\right)$, indicating rapid absorption after oral administration. In the study performed by Zhou et al. the interindividual variability in $\mathrm{CL} / F$ was modest (20\%) [11], but interoccasion variability was not taken into account. In our study, we were able to estimate both interoccasion and interindividual variability in $\mathrm{CL} / F$. The interindividual variability in $\mathrm{CL} / F$ was somewhat higher $(27.4 \%)$ than found by Zhou et al., which may be explained by our use of an unselected cohort.

A small proportion of the population (10.6\%) was observed to be associated with a larger additive error than the rest, which could not be explained by any of the patient characteristics or other identifiable factors.

WT, HCV, and ASAT had a significant relationship with $\mathrm{CL} / F$. Nevirapine is extensively metabolized in the liver by CYP450 enzymes. A value of ASAT $>1.5 \times$ ULN at baseline and coinfection with HCV may reflect hepatic dysfunction, thus resulting in a smaller capacity of the liver to metabolize nevirapine to its inactive metabolites. No effect of renal function on $\mathrm{CL} / F$ was found, which was expected based on the minor role of the kidney in the elimination of nevirapine. Gender was also found to be a significant covariate for $\mathrm{CL} / \mathrm{F}$ when introduced in a univariate manner in the basic model, but not when it was tested in the multivariate analysis. However, the median weight of women and men in this study population differed considerably (58 and $72 \mathrm{~kg}$, respectively). Thus, the lack of significance of gender as a covariate may be explained by a correlation with WT.

Although not significant, patients from the Negroid race appeared to have lower CL/ $F$ than those from other races (data not shown). CYP3A is primarily responsible for metabolism of nevirapine to its metabolites. Ethnic differences in pharmacokinetics have been reported for several drugs [19-24]. Wandel et al. [20] found a significantly lower systemic clearance of midazolam, metabolized by CYP3A, in African Americans when compared with European Americans. The reduction in hepatic CYP3A activity was associated with the possession of a variant $\mathrm{CYP} 3 \mathrm{~A} 4 \star 1 \mathrm{~B}$ allele, which is more frequent in African Americans. Besides genetic factors, environmental factors (e.g. diet) could also contribute to ethnic differences in the pharmacokinetics of nevirapine.

St John's wort has been shown to increase CL/F of nevirapine by $35 \%$, probably by inducing CYP3A [25]. 
Although the effect of St John's wort did not meet our criteria for significance, this earlier finding was compatible with the $21 \%$ increase in $\mathrm{CL} / F$ found in this study.

We and others have described a correlation between the plasma concentration of nevirapine and its efficacy $[9,10]$. In addition, there are indications that a relationship between plasma concentration and toxicity exists. For example, Barreiro et al. demonstrated that the incidence of rash could be diminished by using a slowly escalating dose of nevirapine, (namely $100 \mathrm{mg}$ for the first week, and increasing by $100 \mathrm{mg}$ daily per week until achieving the maximum dose by the fourth week) [26] Relationships between plasma concentration and efficacy and/or toxicity suggest that therapeutic drug monitoring of nevirapine might be useful combined with our model for the Bayesian estimation of individual pharmacokinetic parameters.

In conclusion, a model to describe the pharmacokinetics of nevirapine was developed. For this purpose, we used a patient population attending a routine HIV outpatient clinic. Patient characteristics causing interindividual variability in nevirapine pharmacokinetics were identified, and may lead to a further optimization of nevirapine-containing therapy.

This research was supported by ZAO Health Insurances, Amsterdam, The Netherlands. The authors would like to thank Boehringer Ingelheim for financial support.

\section{References}

1 Murphy RL, Montaner J. Nevirapine: a review of its development, pharmacological profile and potential for clinical use. Exp Opin Invest Drugs 1996; 5: 1183-1199.

2 Viramune Product Monograph. Columbus, Ohio, USA: Roxane Laboratories, Inc.. November 2, 2000.

3 Van Heeswijk RPG, Veldkamp AI, Mulder JW, et al. The steady-state pharmacokinetics of nevirapine during once daily and twice daily dosing in HIV-1-infected individuals. AIDS 2000; 14: F77-F82.

4 Cheeseman SH, Hattox SE, McLaughlin MM, et al. Pharmacokinetics of nevirapine: initial single-rising-dose study in humans. Antimicrob Agents Chemother 1993; 37: 178-182.

5 Erickson DA, Mather G, Trager WF, et al. Characterization of the in vitro biotransformation of the HIV-1- reverse transcriptase inhibitor nevirapine by human hepatic cytochromes P-450. Drug Metab Dispos 1999; 27: 1488-1495.

6 Katlama C, Murphy R, Johnson V, et al. The Atlantic Study: A randomised open-label study comparing two protease inhibitors (PI) -sparing antiretroviral stategies versus a standard PI-containing regimen, 48 week data. 39th Interscience Conference on Antimicrobial Agents and Chemotherapy, San Francisco, September 26-29, 1999, LB-22.

7 Montaner JSG, Reiss P, Cooper D, et al. A randomized, double-blind trial comparing combinations of nevirapine, didanosine, and zidovudine for HIV-infected patients. The INCAS Trial. JAMA 1998; 279: 930-937.

8 Jong MD, Vella S, Carr A, et al. High-dose nevirapine in previously untreated human immunodeficiency virus type 1infected persons does not result in sustained suppression of viral replication. J Infect Dis 1997; 175: 966-970.

9 Havlir D, Cheeseman SH, McLaughlin M, et al. High-dose nevirapine: Safety, pharmacokinetics, and antiviral effect in patients with human immunodeficiency virus infection. $J$ Infect Dis 1995; 171: 537-545.

10 Veldkamp AI, Weverling GJ, Lange JMA, et al. High exposure to nevirapine in plasma is associated with an improved virological response in HIV-1-infected individuals. AIDS 2001; 15: 1089-1095.

11 Zhou X-J, Sheiner LB, D'Aquila RT, et al. Population pharmacokinetics of nevirapine, zidovudine, and didanosine in human immunodeficiency virus-infected patients. Antimicrob Agents Chemother 1999; 43: 121-128.

12 Lamson $\mathrm{M}$, Maldonado S, Hutman $\mathrm{H}$, et al. The effects of underlying renal or hepatic dysfunction on the pharmacokinetics of nevirapine (Viramune) [abstract TuPeB3301]. 13th International AIDS Conference, Durban, July 9-14, 2000.

13 Heeswijk RPG, Hoetelmans RMW, Meenhorst PL, et al. Rapid determination of nevirapine in human plasma by ionpair reversed phase high-performance liquid chromatography with ultraviolet detection. J Chromatogr B 1998; 713: 395-399.

14 Beal SL, Sheiner LB. NONMEM User's Guide University of California at San Francisco: NONMEM Project Group, 1998.

15 Johnsson EN, Karlsson MO. Xpose - an S-PLUS based population pharmacokinetic-pharmacodynamic model building aid for NONMEM. Comp Meth Prog Biomed 1998; 58: 51-64.

16 Higaki K, Yamashita S, Amidon GL. Time-dependent oral absorption models. J Pharmacokin Pharmacodyn 2001; 28: 109128.

17 Karlsson MO, Sheiner LB. The importance of modeling interoccasion variability in population pharmacokinetic analyses. J Pharmacokin Biopharm 1993; 21: 735-750.

18 Holford NHG. Wings for NONMEM. http://wfn.sourceforge.net/ July 23, 2001.

19 Shimada T, Yamazaki H, Mimura M, et al. Interindividual variations in human liver cytochrome P-450 enzymes involved in the oxidation of drugs, carcinogens and toxic chemicals: studies with liver microsomes of 30 Japanese and 30 Caucasians. J Pharmacol Exp Ther 1994; 270: 414-423.

20 Wandel C, Witte JS, Hall JM, et al. CYP3A activity in African American and European American men: Population differences and functional effect of the CYP3A4*1B5'promoter region polymorphism. Clin Pharmacol Ther 2000; 68: 82-91.

21 Mancinelli LM, Frassetto L, Floren LC, et al. The pharmacokinetics and metabolic disposition of tacrolimus: a comparison across ethnic groups. Clin Pharmacol Ther 2001; 69: $24-31$.

22 Kinirons MT, Lang CC, He HB, et al. Triazolam pharmacokinetics and pharmacodynamics in Caucasians and Southern Asians: ethnicity and CYP3A activity. Br J Clin Pharmacol 1996; 41: 69-72.

23 Sowunmi A, Rashid TJ, Akinyinka OO, Renwick AG. 
Ethnic differences in nifedipine kinetics: comparisons between Nigerians, Caucasians and South Asians. Br J Clin Pharmaol 1995; 40: 489-493.

24 Dai D, Tang J, Rose R, et al. Identification of variants of CYP3A4 and characterization of their abilities to metabolize testosterone and chlorpyrifos. J Pharmacol Exp Ther 2001; 299: 825-831.
25 De Maat MMR, Hoetelmans RMW, Mathôt RAA, et al. Drug interaction between St John's wort and nevirapine. AIDS 2001; 15: 420-421.

26 Barreiro P, Soriano V, Casas E, et al. Prevention of nevirapine-associated exanthema using slow dose escalation and/or corticosteroids. AIDS 2000; 14: 2153-2157. 